\title{
Non-traumatic Ocular and Periocular Hemorrhages in a Hypertensive Patient under Continuous Ambulatory Peritoneal Dialysis and Warfarin Therapy
}

\author{
Hung-Wen Yen ${ }^{1,2}$, Ling-Ing Lau ${ }^{2,3}$, Wu-Chang Yang ${ }^{1,2}$, Pei-Yu Lin ${ }^{2,4}$, Chia-Lin Shen ${ }^{1,2}$, \\ Fen-Hsiang $\mathrm{Hu}^{5}$ and Chih-Ching $\mathrm{Lin}^{1,2}$
}

\begin{abstract}
We herein present the first reported case of severe proptosis caused by ocular and periocular hemorrhages in a continuous ambulatory peritoneal dilaysis patient without previous history of trauma. The bleeding tendency caused by uremia and the use of warfarin during uncontrolled high blood pressure were most likely responsible for her ocular and periocular hemorrhages. Appropriate control of blood pressure and adequate selfcare education are important for the prevention and treatment of any bleeding complications in uremic patients receiving both maintenance anticoagulation therapy and peritoneal dialysis.
\end{abstract}

Key words: non-traumatic ocular hemorrhage, periocular hemorrhage, sub-tenon hemorrhage, hypertension, continuous ambulatory peritoneal dialysis, warfarin

(Intern Med 53: 2337-2339, 2014)

(DOI: 10.2169/internalmedicine.53.2866)

\section{Introduction}

Ocular and periocular hemorrhages are extremely rare complications in patients undergoing regular dialysis, especially those without a history of surgery or trauma. In this report, we herein describe a case of non-traumatic ocular and periocular hemorrhages in a hypertensive patient under continuous ambulatory peritoneal dialysis and warfarin therapy.

\section{Case Report}

A 43-year-old anuric woman undergoing regular continuous ambulatory peritoneal dialysis (CAPD) for chronic glomerulonephritis-related end-stage renal disease was hospitalized due to the gradual onset of a protruding left eyeball with poor vision in her left eye for two weeks. She had chronic shortness of breath and dry cough resulting from severe anemia and fluid overload due to poor compliance to our recommendation of salt and water restriction. She denied any history of trauma to her left eye during the two weeks prior to this admission. She was taking warfarin (2.5 $\mathrm{mg}$ once daily) as a part of maintenance anticoagulation therapy after a mitral valve replacement for mitral valve regurgitation due to lower prothrombin time (INR) values (0.94-1.09) in the two months prior to this admission. However, her blood pressure was not adequately controlled for several weeks because of her poor compliance to antihypertensive agents and peritoneal dialysis. Her initial vital signs on admission were blood pressure 203/99 $\mathrm{mmHg}$, heart rate $102 / \mathrm{min}$, respiratory rate $20 / \mathrm{min}$, and body temperature $36.8^{\circ} \mathrm{C}$. Her laboratory data on admission included hemoglobin $5.9 \mathrm{~g} / \mathrm{dL}$, leukocyte $6,300 / \mathrm{mm}^{3}$, platelet $505,000 / \mathrm{mm}^{3}$, blood urea nitrogen $93 \mathrm{mg} / \mathrm{dL}$, serum creatinine $13.48 \mathrm{mg} / \mathrm{dL}$, calcium $9.3 \mathrm{mg} / \mathrm{dL}$, phosphate $9.6 \mathrm{mg} /$ $\mathrm{dL}$, and serum $\beta 2$-microglobulin $116.2 \mathrm{mg} / \mathrm{L}$. Her indices of CAPD therapy were weekly Kt/V 2.05 and weekly creatinine clearance (WCC) $39.6 \mathrm{~L} /$ week $/ 1.73 \mathrm{~m}^{2}$ body surface area 2 months before this admission. The reason for inade-

\footnotetext{
${ }^{1}$ Division of Nephrology, Department of Medicine, Taipei Veteran General Hospital, Taiwan, ${ }^{2}$ School of Medicine, National Yang-Ming University, Taiwan, ${ }^{3}$ Department of Ophthalmology, Taipei Veterans General Hospital, Taiwan, ${ }^{4}$ Department of Family Physician, Taipei Veteran General Hospital, Taiwan and ${ }^{5}$ Division of Nephrology, Department of Medicine, Wei-Gong Memorial Hospital, Taiwan

Received for publication March 11, 2014; Accepted for publication April 9, 2014

Correspondence to Dr. Chih-Ching Lin, lincc2@vghtpe.gov.tw
} 


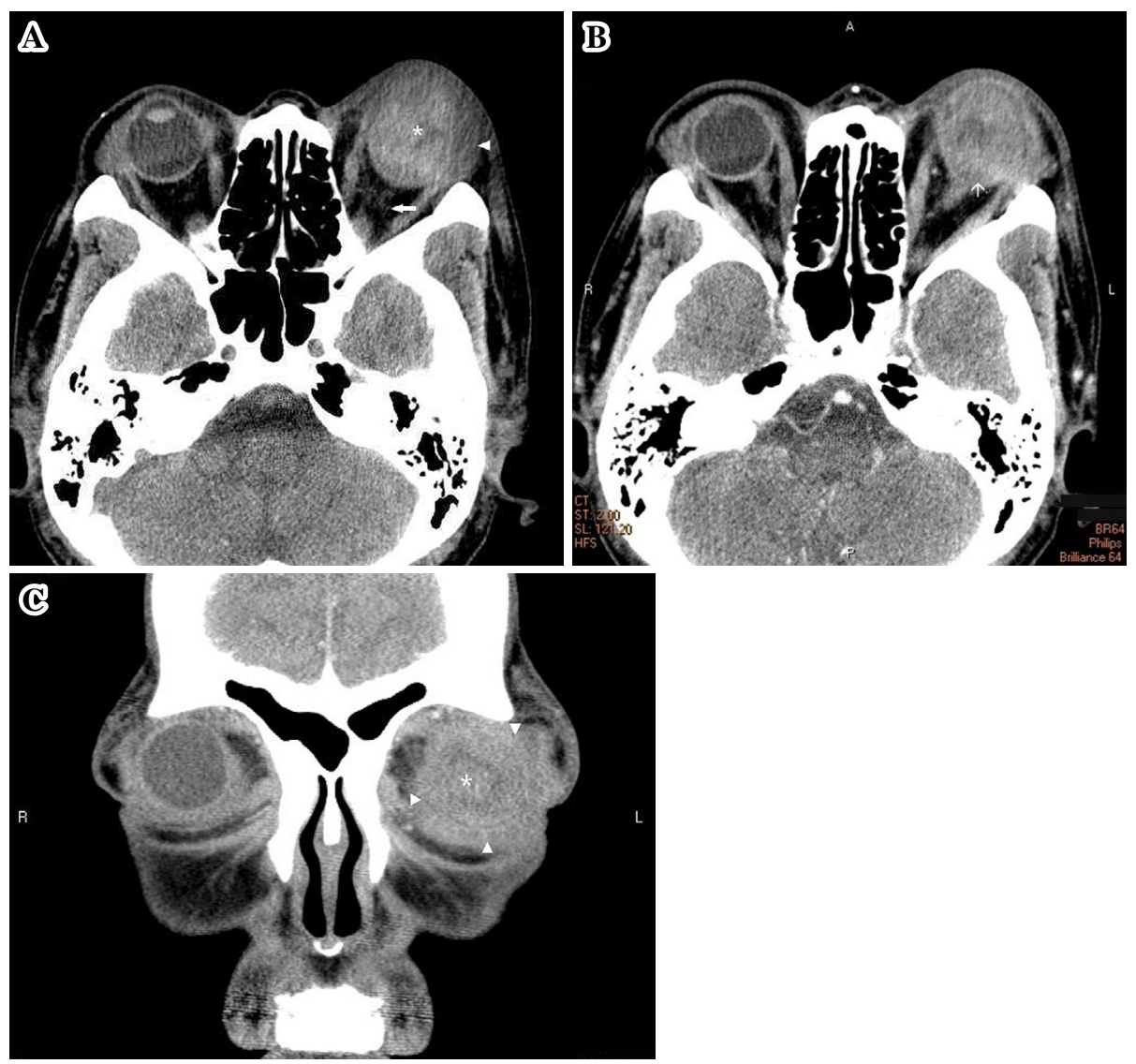

Figure. (A) The transverse view of the orbital CT shows a retrobulbar hematoma (arrow); subtenon and subconjunctival hematoma (arrowhead); and intraocular hematoma (asterisk). (B) The transverse view of the orbital CT shows a posterior sub-tenon hematoma (arrow). (C) The coronal view of the orbital CT shows a sub-tenon and subconjunctival hematoma (arrowheads) and an intraocular hematoma (asterisk).

quate dialysis was most likely her noncompliance to CAPD therapy. She was only exchanging 6-8 L dialysate per day despite of our prescription of dialysate exchange up to $10 \mathrm{~L}$ daily.

Contrast-enhanced computed tomography (CT) of the orbital cavity was then performed and showed several heterogeneous hematomas over retrobulbar, intraocular, sub-tenon, and subconjunctival areas of the left eyeball (Figure). Rupture of the ciliary artery was then highly suspected because it is the major source of blood supply to the sub-tenon area, thus leading to the simultaneous, very extensive hemorrhage in the ocular and periocular areas. An ophthalmologist was consulted but the visual loss of her left eye was irreversible because she had not sought medical assistance early enough. Surgical removal of the affected eyeball was suggested but she refused. Thus, only conservative treatment could be given for the treatment of her catastrophic visual loss. After admission, she exchanged $10 \mathrm{~L}$ dialysate daily according to our prescription, and her blood urea nitrogen was improved to $75 \mathrm{mg} / \mathrm{dL}$, serum creatinine to $9.59 \mathrm{mg} / \mathrm{dL}, \mathrm{Kt} / \mathrm{V}$ to 2.22 , and WCC to $49.9 \mathrm{~L} /$ week $/ 1.73 \mathrm{~m}^{2}$ body surface area on day 11 of admission. Her proptosis and intraocular hematoma resolved gradually in the following 4 weeks due to adequate CAPD therapy as well as better control of her blood pres- sure and judicious titration of the dose of anticoagulant therapy; however, her visual loss was irreversible. The patient was then discharged and her status remained unchanged for a year following routine follow-ups.

\section{Discussion}

To the best of our knowledge, this is the first reported case of severe proptosis caused by an ocular and periocular hemorrhages in a CAPD patient without previous history of trauma. Several cases of retrobulbar hemorrhage (1) or suprachoroidal hemorrhage (2) following an eye trauma or an optic surgery were reported in patients undergoing hemodialysis or peritoneal dialysis. However, it is rare for dialysis patients to suffer from ocular and periocular hemorrhages in the absence of surgery or trauma. Similarly, Tajika et al. also reported choroidal hemorrhaging without proptosis as a complication following an episode of cough in a patient with hypertension and chronic renal failure but without history of trauma or surgery (3). In our case, the bleeding tendency caused by uremia and the use of warfarin during uncontrolled high blood pressure were most likely responsible for her ocular and periocular hemorrhages. It has been reported that hemodialysis patients under warfarin treatment 
may have a higher risk for major bleeding episodes and inter-measurement INR variability compared with nonhemodialysis patients under warfarin treatment or hemodialysis patients without warfarin treatment (4). We infer that the gradual onset of her blurred vision and proptosis may possibly result from the slowly-developed blood leak and hematoma formation caused by her bleeding tendency, although a retrobulbar hematoma usually occurs abruptly in a traumatic patient.

Ocular hemorrhaging (this case) and intracranial hemorrhaging $(\mathrm{ICH})$ in uremic patients may share some common mechanisms of major bleeding, including hypertension, higher level of uremic toxins due to inadequate dialysis, platelet dysfunction, and the use of anticoagulation. In addition to the previously mentioned common mechanisms for severe hemorrhaging in uremic patients, however, mitral valve regurgitations may increase the pulmonary venous pressure, thus leading to chronic cough associated with the simultaneously strenuous Valsalva maneuver. Elevated intraocular pressure caused by chronic kidney disease and chronic cough may also contribute to this complication $(5,6)$. Furthermore, pre-existent vascular risk factors, such as severe hypertension and hypercholesterolemia, may also result in endothelial dysfunction. These two specific mechanisms (i.e., a higher intraocular pressure and endothelial dysfunction) may also play important roles as predisposing risk factors in the development of the ocular and periocular hemorrhages in this case.

The spontaneous resolution of proptosis and the ocular and periocular hematoma in this patient may result from the absence of anticoagulation as well as the relatively stable hemodynamic condition during renal replacement therapy by peritoneal dialysis rather than by hemodialysis. This could be advantageous for the treatment of any bleeding complication in uremic patients under peritoneal dialysis. In summary, good control of blood pressure and adequate self-care education are important for the prevention and treatment of any bleeding complications including ocular or periocular hemorrhaging in uremic patients receiving both maintenance anticoagulation therapy and peritoneal dialysis, a choice for renal replacement therapy with a much lower bleeding risk than hemodialysis.

The authors state that they have no Conflict of Interest (COI).

\section{References}

1. Lee KY, Tow S, Fong KS. Visual recovery following emergent orbital decompression in traumatic retrobulbar haemorrhage. Ann Acad Med Singapore 35: 831-832, 2006.

2. Kim M, Lee SC, Lee SJ. Abrupt spontaneous suprachoroidal hemorrhage post-23-gauge vitrectomy during peritoneal dialysis. Clin Ophthalmol 7: 1175-1179, 2013.

3. Tajika T, Yokozeki H, Ishimaru K, Naito T, Shiota H. Rare case of choroidal hemorrhage complicated with hypertension due to chronic renal failure. J Med Invest 55: 151-155, 2008.

4. Phelan PJ, O'Kelly P, Holian J, et al. Warfarin use in hemodialysis patients: what is the risk? Clin Nephrol 75: 204-211, 2011.

5. Lewis CD, Perry JD. Retrobulbar hemorrhage. Expert Rev Ophthalmol 2: 557-570, 2007.

6. Nongpiur ME, Wong TY, Sabanayagam C, Lim SC, Tai ES, Aung T. Chronic kidney disease and intraocular pressure: the Singapore Malay Eye Study. Ophthalmology 117: 477-483, 2010.

(C) 2014 The Japanese Society of Internal Medicine http://www.naika.or.jp/imonline/index.html 